\title{
Continuous subcutaneous insulin infusion versus multiple dose insulin
}

\author{
Yashdeep Gupta ${ }^{1}$, Sanjay Kalra ${ }^{2}$ \\ Sri Lanka Journal of Diabetes, Endocrinology and Metabolism 2014; 4: 22-29
}

\begin{abstract}
Continuous subcutaneous insulin infusion (CSII), also known as insulin pump therapy and multiple dose insulin (MDI) are the two main intensified insulin regimens that have been used to achieve strict glycaemic control in patients with diabetes mellitus, especially with type 1 diabetes (T1DM). Each mode of therapy has its own merits and demerits. CSII is a costly and complex therapeutic intervention, but on the other hand provides flexibility, improves patient's quality of life, glycaemic control and decreases hypoglycaemic episodes in certain situations. This review will summarize current evidence on CSII vs MDI on various outcomes. This will help health professionals to select the most appropriate method of insulin administration for the given situation.
\end{abstract}

Keywords: continuous subcutaneous insulin infusion, CSII, insulin pump therapy, multiple dose insulin, MDI, diabetes men

\section{Introduction}

Suboptimal glycemic control in individuals with diabetes leads to chronic complications and increases mortality. Therefore, efficient glucose control is essential for the prevention of life threatening complications of the disease. The increasing need of aggressive diabetes treatment has led to the improvement of insulin therapy and its implementation techniques. CSII and MDI regimens are the two main intensified insulin regimens. The development of CSII by insulin pumps and shortacting insulin analogues are innovations designed to improve glycaemic control and quality of life (QOL), while limiting adverse effects, such as hypoglycaemia. They represent important advances in the treatment of diabetes.

CSII, also known as insulin pump therapy, uses a small, portable electromechanical pump to infuse shortacting insulin via a subcutaneously implanted cannula to provide basal delivery, with patient-activated prandial boluses. MDI, also known as basal-bolus therapy, is a regimen that employs long-acting insulin formulations (isophane, glargine, detemir) to supply the basal component, with rapid acting insulin (aspart, lispro, glulisine) or short acting insulin (regular insulin) to supply the boluses.

The insulin pump therapy is an expensive addition in the management of patients with diabetes. Therefore, it is important to understand whether CSII provides additional benefits in terms of glycaemic control, hypoglycaemic episodes, quality of life and certain other parameters as compared to MDI. We summarize available data mainly from latest meta-analysis that compare pumps with multiple dose insulin injections. The review will address the issues separately on type 1 diabetes mellitus in children, type 1 diabetes mellitus in adults, type 2 diabetes mellitus and pregnancy. This will be helpful in providing objective information to health professionals when making decisions about the use of CSII in clinical practice.

Search strategy: Pubmed with "Insulin pump therapy” or "Continuous subcutaneous insulin infusion” or "Insulin analogues”. Limits Meta-analysis, Reviews, Systematic reviews, Randomised control trials, Guidelines, English language, 10 years. Additional relevant cross references were retrieved.

\section{Type 1 diabetes mellitus (adults)}

HbA1c

A recent meta-analysis compared rapid-acting analogue-based CSII with MDI (rapid-acting analogues with neutral protamine Hagedorn/long acting analogue insulin) (1). It found that CSII decreased HbA1c levels more than MDI did, combined mean between-group difference was $-0.30 \%$. However, the pooled estimate was influenced by 1 study in which participants had a higher HbA1c level at enrolment (9.3\%) compared with that of the other studies (7.7\% to $8.2 \%$ ), resulting in greater

${ }^{1}$ DM Endocrinology, Assistant Professor (Endocrinology), Department of Medicine Government Medical College and Hospital Sector 32 Chandigarh 160032, India, ${ }^{2} D M$ Endocrinology, Department of Endocrinology, Bride Hospital Karnal Haryana, India. 
opportunity for a large decrease in HbA1c levels in that study $(-0.84 \%)$ than in the other studies $(-0.1 \%$ to $0.25 \%)$. The difference between CSII and MDI became null (combined mean between-group difference, -0.01\%) after this study was removed. Furthermore, the study with largest effect used NPH insulin as basal insulin (2). The studies which used glargine as basal insulin in addition to rapid acting analogues found difference of $\mathrm{HbA} 1 \mathrm{C}$ of only $-0.1 \%$ (statistically insignificant in both studies) $(3,4)$. The results of this meta-analysis implies that in adults with type 1 diabetes mellitus, CSII shows favourable effects on glycaemic control (greater decrease in HbA1C levels), in participants who had higher HbA1C levels at enrolment than who had closer to the target HbA1C level at enrolment. The insulin pump therapy using rapid acting analogues in comparison to MDI (rapid acting analogues + long acting analogue) had clinically insignificant reduction in HbA1C (1).

\section{Daily mean blood glucose}

In 10 studies that included participants more than 18 years of age the mean difference of daily mean blood glucose was estimated to be $-18 \mathrm{mg} / \mathrm{dl}$ (95\% CI -27 to -9) in favour of CSII compared with MDI (5).

\section{Hypoglycaemic events}

There was a similar incidence rate of severe hypoglycaemia in the 2 intervention groups (CSII and MDI). The incidence of mild hypoglycaemia and nocturnal hypoglycaemia was also similar (1). This meta-analysis compared CSII using insulin analogues with MDI in which the pre-prandial insulin was also insulin analogue (1).

\section{Quality of life}

A meta-analysis of 2 studies favoured CSII over MDI for diabetes mellitus specific QOL (1). There was also improvement in general QOL between the 2 intervention groups favouring CSII.

\section{Weight gain}

Weight gain did not differ between CSII and MDI on meta-analysis of 4 studies (1).

\section{Adverse events}

Overall, information on adverse treatment effects other than hypoglycaemia is insufficient (6). As per analysis of quality RCTs in Cochrane Review, none of the studies reported on mortality, morbidity or costs (5).

\section{Guidelines recommendations}

In the United Kingdom, the National Institute for Health and Clinical Excellence (NICE) has recommended
CSII as a cost-effective treatment option in adults with T1DM when attempts to achieve target HbA1c levels with MDI have resulted in disabling hypoglycaemia or when HbA1c levels have remained $\geq 8.5 \%$ despite best efforts (7). As per American Association of Clinical Endocrinologists (AACE) insulin pump management task force, patients with type $1 \mathrm{DM}$ who do not reach glycaemic goals despite adherence to a maximum MDI, are candidates for CSII (8), especially if they have:

i. Very labile DM (erratic and wide glycaemic excursions, including recurrent DKA.

ii. Frequent severe hypoglycaemia and/or hypoglycaemia unawareness.

iii. Significant “dawn phenomenon”, extreme insulin sensitivity.

The information on various outcomes by using CSII, as compared to MDI in T1DM (adults) is summarized in Table 1.

\section{Type 1 DM (Children)}

\section{HbA1c}

Meta-analysis on 7 RCTs (16 or more weeks of followup), compared MDI with CSII in children and adolescents with type 1 diabetes mellitus (1). No difference was found between groups in HbA1c level as compared to baseline. Results were similar among adolescents older than 12 years (combined mean between group difference in change from baseline in HbA1c, $-0.10 \%$ [95\% CI, $-0.48 \%$ to $0.27 \%$ ]; and less precise among children aged 12 years or younger (combined mean between-group difference in change from baseline in HbA1c, $-0.05 \%$ [CI, $-1.01 \%$ to $0.96 \%$ ]. The metaanalysis concluded that CSII and MDI have similar effects on HbA1c in children and adolescents with type 1 diabetes mellitus.

\section{Daily mean blood glucose}

In 4 studies that included participants less than 18 years of age the mean difference of daily mean blood glucose was estimated to be $-4 \mathrm{mg} / \mathrm{dl}$ (95\% CI -14 to 7) in favour of CSII compared with MDI. The difference was not statistically significant (5).

\section{Hypoglycaemic events}

Similar rates of severe hypoglycaemia were found in the 2 intervention groups (CSII and MDI) (1). The risk reduction favoured CSII, although the comparison was not statistically significant. Results were similar in metaanalysis of 3 RCTs in adolescents and of 2 RCTs in children aged 12 years or younger. CSII and MDI had similar effects on nocturnal hypoglycaemia. The meta-analysis concluded that CSII and MDI have similar effects on the incidence of severe hypoglycaemia in children and adolescents with type 1 diabetes mellitus. 
Table 1. Effect on outcomes with CSII in comparison to MDI in adults with T1DM

Outcome Which is better? CSII or MDI

HbA1c

Daily mean blood glucose

Hypoglycaemic events
Quality of life
Weight gain

Adverse events

\section{Guidelines recommendations}

CSII group had more reduction in HbA1C (Clinically insignificant).

CSII showed greater decrease in HbA1C levels in participants who had higher HbA1C levels at enrolment.

The mean difference of daily mean blood glucose was estimated to be $-18 \mathrm{mg} /$ $\mathrm{dl}$ in favour of CSII compared with MDI.

\section{No difference}

There is improvement in general quality of life with CSII.

No difference

Insufficient information

- National Institute for Health and Clinical Excellence (NICE) has recommended CSII as a cost-effective treatment option in adults with T1DM when attempts to achieve target HbA1C levels with MDI have resulted in disabling hypoglycaemia or when HbA1C levels have remained $\geq 8.5 \%$ despite best efforts.

- As per American Association of Clinical Endocrinologists insulin pump management task force patients with type $1 \mathrm{DM}$ who do not reach glycaemic goals despite adherence to a maximum MDI, are candidates for CSII especially if they have:

i. Very labile DM (erratic and wide glycaemic excursions, including recurrent DKA

ii. Frequent severe hypoglycaemia and/or hypoglycaemia unawareness

iii. Significant “dawn phenomenon”, extreme insulin sensitivity

\section{Quality of life}

A pooled analysis of 2 studies showed no significant difference in general QOL questionnaire (1). A metaanalysis that examined the metabolic and psychosocial impact of CSII and included five paediatric studies reported no consistent differences in anxiety, depression, QOL, self-esteem, and family functioning (9). In qualitative studies using standardized interview techniques, on switching from MDI to CSII, parents of infants and toddlers reported more freedom, flexibility, and spontaneity in their lives as well as reduced parental stress and worry regarding their child's overall care (10).

\section{Weight gain}

A pooled analysis of 2 studies shows no betweengroup mean difference in weight (1).

\section{Adverse events}

None of the studies reported on mortality, morbidity or costs in studies analysed in Cochrane review (5). Individuals using CSII are potentially at increased risk of developing diabetic ketoacidosis (DKA), with DKA rates varying from 2.7 to 9 episodes per 100 patient-years (11). However, as with MDI, DKA is preventable in CSII using published DKA prevention guidelines (12). In Norwegian children with diabetes, the nationwide incidence of DKA (approximately 4 episodes per 100 patient-years) did not change despite an increase in CSII use from 5\% in 2001 to $38 \%$ in 2005 (13).

\section{Guidelines recommendations}

As per NICE guidelines (7), CSII is recommended as a treatment option when MDI is considered impractical or inappropriate in children $<12$ years with T1DM, and with the expectation that children would normally undergo a trial of MDI between the ages of 12 and 18 years. CSII should be discontinued (in adults and children $\geq 12$ years who have been started on CSII because of elevated HbA1C or disabling hypoglycaemia) if no sustained improvement 
in HbA1c or rate of hypoglycaemic episodes occurs. In children $\geq 12$ years with T1DM, CSII is recommended as a treatment option when attempts to achieve target HbA1c levels with MDI have resulted in disabling hypoglycaemia or when $\mathrm{HbA1c}$ levels have remained high ( $\geq 8.5 \%$ ) despite a high level of care (7).

As per joint consensus statement from the European Society for Paediatric Endocrinology (ESPE), The Lawson Wilkins Paediatric Endocrine Society (LWPES), and the International Society for Paediatric and Adolescent Diabetes (ISPAE) (endorsed by the American Diabetes Association and the European Association for the Study of Diabetes) (12), all paediatric patients with type 1 diabetes are potential candidates for CSII, and there is no lower age limit for initiating CSII. CSII should be considered in the conditions listed below:

1. Recurrent severe hypoglycaemia

2. Wide fluctuations in blood glucose levels regardless of A1C

3. Suboptimal diabetes control (i.e., A1C exceeds target range for age)

4. Microvascular complications and/or risk factors for macrovascular complications

5. Good metabolic control but insulin regimen that compromises lifestyle

The information on various outcomes by using CSII, as compared to MDI in T1DM (children and adolescents) is summarized in Table 1.

\section{Type 2 DM (adults)}

\section{HbA1c}

A meta-analysis on 4 RCTs, compared CSII with MDI in adults with type 2 diabetes mellitus. CSII used rapidacting analogue. MDI was based on long-acting analogues with rapid-acting analogues or NPH with rapid-acting analogue/regular insulin in the MDI group (1). This metaanalysis found that CSII decreased HbA1c levels more than MDI did, (combined mean between-group difference was $-0.18 \%$ [CI, $-0.43 \%$ to $0.08 \%$ ] ). The results were not statistically significant, neither have they indicated any clinical advantage of CSII over MDI.

\section{Hypoglycaemic events}

Analysis of findings of two RCTS found a similar rate of severe hypoglycaemia in the 2 intervention groups (CSII and MDI). The incidence of mild hypoglycaemia was also similar (1).

\section{Quality of life}

No difference in general QOL or diabetes mellitusspecific QOL between the CSII and MDI intervention groups has been reported (1).

\section{Weight gain}

Weight gain did not differ between CSII and MDI groups in a meta-analysis of 2 studies (1).

\section{Guidelines recommendation}

As per NICE guidance, CSII is not generally recommended in type 2 diabetes mellitus, although some subgroups may benefit (7). The AACE recommends CSII in selected patients with insulin requiring type $2 \mathrm{DM}$ who satisfy any or all of the following (8):

1. C-peptide positive but with suboptimal control on a maximal program of basal/bolus injections

2. Substantial "dawn phenomenon"

3. Erratic lifestyle (eg, frequent long distance travel, shift-work, unpredictable schedules leading to difficulty maintaining timing of meals)

4. Severe insulin resistance, candidate for U500 insulin by CSII

5. Selected patients with other DM types (eg, post pancreatectomy)

The information on various outcomes by using CSII, as compared to MDI in T2DM is summarized in Table 3.

\section{Pregnancy}

The number of randomized controlled trials comparing CSII and MDI in pregnancy is scarce and the available studies have included a small number of participants (14). A majority of the studies conducted in pregnant women are observational in nature involving both type 1 and type 2 diabetics (15). Most of the results are from trials in the 1980s and early 90s when pumps were less reliable and less technically sophisticated (16). These used regular insulin, instead of newer insulin analogues that are available now and are the standard of care. Therefore, the comparison between the CSII and MDI, presented below has to be interpreted keeping these caveats in mind.

\section{HbA1c}

In Cochrane review, only one RCT fulfilled the eligibility criteria. In this RCT, HbA1c was compared in 16 patients in each arm and in each trimester. There was no statistically significant difference in mean HbA1c in the two arms in any of the trimester. The mean difference in $\mathrm{HbA} 1 \mathrm{c}$ was $0.2 \%$ in first trimester favouring CSII and was $0.7 \%$ and $0.1 \%$ favouring MDI in second and third trimester respectively (14). In another meta-analysis, the three studies were included (selection criteria for trials in this meta-analysis were not stringent). The meta-analysis performed on glycosylated hemoglobin at term in 3 studies showed no significant differences between the 2 treatment groups at any period of time (15). 
Table 2. Effect on outcomes with CSII in comparison to MDI in children and adolescents with T1DM

\begin{tabular}{ll}
\hline Outcome & Which is better? CSII or MDI \\
\hline HbA1c & No difference \\
Daily mean blood glucose & No difference \\
Hypoglycaemic events & No difference \\
Quality of life & No difference in general QOL score \\
Weight gain & No difference \\
Adverse events & No difference (DKA episodes) \\
Guidelines recommendations & NICE guidance
\end{tabular}

- CSII is recommended as a treatment option when MDI is considered impractical or inappropriate in children $<12$ years with T1DM

- CSII is recommended as a treatment option when attempts to achieve target HbA1c levels with MDI have resulted in disabling hypoglycaemia or when HbA1c levels have remained high ( $\geq 8.5 \%$ ) despite a high level of care.

ISPAD, LWPES, ESPE recommendations

CSII should be considered in the conditions listed below:

- Recurrent severe hypoglycaemia

- Wide fluctuations in blood glucose levels regardless of A1C

- Suboptimal diabetes control (i.e., A1C exceeds target range for age)

- Microvascular complications and/or risk factors for macrovascular complications

- Good metabolic control but insulin regimen that compromises lifestyle

Table 3. Effect on outcomes with CSII in comparison to MDI in T2DM

\begin{tabular}{|c|c|}
\hline Outcome & Which is better? CSII or MDI \\
\hline HbA1c & No difference \\
\hline Hypoglycaemic events & No difference \\
\hline Quality of life & No difference \\
\hline Weight gain & No difference \\
\hline \multirow[t]{5}{*}{ Guidelines recommendations } & $\begin{array}{l}\text { The AACE recommends CSII in selected patients with insulin requiring type } \\
2 \text { DM who satisfy any or all of the following: }\end{array}$ \\
\hline & $\begin{array}{l}\text { 1. C-peptide positive but with suboptimal control on a maximal program of } \\
\text { basal/bolus injections }\end{array}$ \\
\hline & 2. Substantial “dawn phenomenon” \\
\hline & $\begin{array}{l}\text { 3. Erratic lifestyle (eg, frequent long distance travel, shift-work, } \\
\text { unpredictable schedules leading to difficulty maintaining timing of meals) }\end{array}$ \\
\hline & $\begin{array}{l}\text { 4. Severe insulin resistance, candidate for U500 insulin by CSII. Selected } \\
\text { patients with other DM types (eg, postpancreatectomy) }\end{array}$ \\
\hline
\end{tabular}




\section{Maternal 24 hour mean blood glucose (mg/dl)}

The meta-analysis of 3 studies revealed insignificant $(0.12 \mathrm{mg} / \mathrm{dl})$ difference in maternal 24 hour mean blood glucose in first trimester between the two arms. The difference was $1.77 \mathrm{mg} / \mathrm{dl}$ and $0.08 \mathrm{mg} / \mathrm{dl}$ in second and third trimester respectively, all favouring MDI (14).

\section{Maternal hypoglycaemia}

In a meta-analysis by Mukhopadhay et al, relative to women allocated to MDI therapy, women who received CSII had more hypoglycaemic spells (22.3\% vs $19.2 \%$; OR, 1.34, $\mathrm{p}=0.46$ ). This meta-analysis was reported on 5 studies (15). In another meta-analysis, the risk ratio was 3.0 with more hypoglycaemic events in CSII than in MDI. The number of hypoglycaemic events was only 3 in 30 total patients on CSII as compared to in 1 patient out of 31 patients in MDI group (14).

\section{Neonatal hypoglycaemia}

Neonatal hypoglycaemia was reported from 5 studies in a meta-analysis. There was no significant difference between the two treatment groups (19.1\% in CSII vs 14.8\% in MDI group; OR, 1.31, $\mathrm{P}=0.51$ ) (15).

\section{Ketoacidosis}

The meta-analysis of 4 studies, (published from 1986 to 1993), have shown more episodes of ketoacidosis in CSII group (5 episodes in pooled 70 subjects in CSII group as compared to none in 73 subjects in MDI group, $\mathrm{p}=0.23)(15)$.

\section{Caesarean rates}

Caesarean section rates did not differ between the CSII and MDI groups (51\% vs 43\%; OR, 1.39, $\mathrm{p}=0.27$ ) in a meta-analysis of 5 studies (15).

\section{Perinatal mortality}

There were 6 stillbirths in CSII group ( $n=94)$ and 1 in MDI group $(n=88)$ in pooled analysis of 5 studies. The OR being 2.5 , but was not statistically significant $(p=0.25)(15)$.

Table 4. Effect on outcomes with CSII in comparison to MDI in pregnancy

\begin{tabular}{|c|c|}
\hline Outcome & Which is better? CSII or MDI \\
\hline HbA1c & No significant difference \\
\hline $\begin{array}{l}\text { Maternal } 24 \text { hour mean } \\
\text { blood glucose (mg/dl) }\end{array}$ & No significant difference \\
\hline Maternal hypoglycaemia & Relatively more with CSII \\
\hline Neonatal hypoglycaemia & No significant difference \\
\hline Ketoacidosis & Relatively more with CSII \\
\hline Caesarean rates & No significant difference \\
\hline Perinatal mortality & Relatively more with CSII \\
\hline $\begin{array}{l}\text { Neonatal birth weight, small or } \\
\text { large for gestational age }\end{array}$ & No significant difference \\
\hline Guidelines opinion & $\begin{array}{l}\text { - As per NICE guidance, CSII should be considered in pregnancy or pre- } \\
\text { conceptually in women with T1DM when the target HbA1c (normally } \\
\leq 6.1 \% \text { ) in the first trimester or pre-conceptually cannot be achieved } \\
\text { without disabling hypoglycaemia. } \\
\text { - As per AACE, the literature does not suggest clear evidence that insulin } \\
\text { pumps are necessary for optimal treatment of women with type } 1 \mathrm{DM} \\
\text { during pregnancy. A robust randomized trial, adequately powered to } \\
\text { assess efficacy out-comes for CSII vs MDI in pregnant women with DM, } \\
\text { is needed. } \\
\text { AACE opines that insulin pump therapy seems to be safe and effective } \\
\text { for maintaining glycaemic control in pregnancies complicated by } \\
\text { gestational DM/type } 2 \text { DM requiring large doses of insulin. }\end{array}$ \\
\hline
\end{tabular}




\section{Neonatal birth weight, small or large for gestational age}

There was no significant difference in the birth weight, rates for small for gestational age or large for gestational age between the two treatment groups (15).

\section{Guidelines recommendations}

As per NICE guidance, CSII should be considered in pregnancy or pre-conceptually in women with T1DM when the target HbA1c (normally $\leq 6.1 \%$ ) in the first trimester or pre-conceptually cannot be achieved without disabling hypoglycaemia (7). As per AACE, the literature does not suggest clear evidence that insulin pumps are necessary for optimal treatment of women with type $1 \mathrm{DM}$ during pregnancy. A robust randomized trial, adequately powered to assess efficacy outcomes for CSII vs MDI in pregnant women with DM, is needed. On the basis of one study, AACE opines that insulin pump therapy seems to be safe and effective for maintaining glycaemic control in pregnancies complicated by gestational DM/type $2 \mathrm{DM}$ requiring large doses of insulin (17).

The information on various outcomes by using CSII, as compared to MDI in pregnancy is summarized in Table 4.

Apart from the specific points that were discussed under different groups of diabetes, there are some other important considerations. Glycaemic variability is a major frustration for patients with T1DM. High glycaemic variability correlates with an increased frequency of hypoglycaemia and individuals with the most variability maintain the highest HbA1c level on MDI, probably to avoid increasing the frequency of hypoglycaemia. CSII reduces both the within-day and day-to-day variability (16). This improvement is probably because the large variation in subcutaneous absorption associated with large injected volumes of long-acting insulin ( $\pm 50 \%$ for isophane) is reduced to about $\pm 3 \%$ with CSII (probably because there is a subcutaneous insulin depot of only about 1 unit at any time during basal rate infusion) (18). The frequency of diabetic ketoacidosis is not significantly different during modern CSII versus MDI; however, the potential risk of ketoacidosis is greater with CSII in the event of pump malfunction with interrupted insulin delivery, or with the increased insulin requirements of illness, because of the smaller subcutaneous insulin depot with CSII.

The selection of an optimal candidate for this complex therapy is important. Clearly, CSII is not appropriate for every patient with insulin-requiring DM. The ideal CSII candidate would be a patient with type $1 \mathrm{DM}$ or absolutely insulin-deficient type $2 \mathrm{DM}$ who currently performs 4 or more insulin injections and 4 or more self-monitored blood glucose measurements daily, is motivated to achieve tighter blood glucose control, is willing, and intellectually and physically able to undergo the rigors of insulin pump therapy initiation and maintenance. Eligible patients should be capable of self-management through frequent selfmonitored blood glucose measurements. Further, candidates must be able to master carbohydrate counting and insulin correction and adjustment formulas and must be prepared to troubleshoot problems related to pump operation and blood glucose levels. Last, patients should be emotionally mature, with a stable life situation, and willing to maintain frequent contact with members of their health care team, in particular their pump-supervising physician. Monomeric, rapid-acting insulin analogues (aspart, lispro, or glulisine) are now considered to be the insulin of choice for pumps (19). Insulin aspart is approved for use in children 2 years and above. Lispro and Glulisine are approved in children above 3 and 4 years respectively. Insulin aspart and lispro are approved for use in pregnancy.

\section{Conclusions}

MDI regimen that includes frequent self-monitoring of blood glucose levels and structured diabetes education can achieve good glycaemic control in many individuals with T1DM. A trial of CSII is indicated in patients who do not achieve acceptable glycaemic control with MDI because of continued, elevated HbA1c levels or disabling hypoglycaemic episodes. Continuous subcutaneous insulin infusion (CSII) can reduce HbA1c levels and hypoglycaemia in many adult patients with type 1 diabetes mellitus (T1DM), compared with multiple daily insulin injections (MDI). The greatest reduction in HbA1c levels with CSII occurs in patients with T1DM who have the worst glycaemic control with MDI. Because of the curvilinear relationship between HbA1c level and microvascular risk, reductions in HbA1c level from this high starting point produce a more marked reduction in the risk of diabetic complications and better costeffectiveness than that achievable for unselected patients with a lower mean HbA1c level. NICE in the UK judged that, when quality of life improvements are taken into account along with reductions in HbA1c level, CSII is cost-effective when HbA1c levels on MDI are $\geq 8.5 \%$ and, therefore, used this cut-off value in their guidance. Blood glucose variability and treatment satisfaction are also usually improved with CSII versus MDI. CSII is not generally recommended in type 2 diabetes mellitus, although some subgroups may benefit. CSII is safe and effective in pregnancy, but no convincing evidence exists from either observational studies or the relatively few RCTs conducted that glycaemic control or pregnancy outcomes differ between CSII and MDI. 


\section{References}

1. Yeh HC, Brown TT, Maruthur N, Ranasinghe P, Berger Z, Suh YD, et al. Comparative effectiveness and safety of methods of insulin delivery and glucose monitoring for diabetes mellitus: a systematic review and meta-analysis. Ann Intern Med 2012; 157: 336-47.

2. DeVries JH, Snoek FJ, Kostense PJ, Masurel N, Heine RJ. Dutch Insulin Pump Study Group. A randomized trial of continuous subcutaneous insulin infusion and intensive injection therapy in type 1 diabetes for patients with longstanding poor glycemic control. Diabetes Care 2002; 25: 2074-80.

3. Bolli GB, Kerr D, Thomas R, Torlone E, Sola-Gazagnes A, Vitacolonna E, et al. Comparison of a multiple daily insulin injection regimen (basal once-daily glargine plus mealtime lispro) and continuous subcutaneous insulin infusion (lispro) in type 1 diabetes: a randomized open parallel multicenter study. Diabetes Care 2009; 32: 1170-6.

4. Thomas RM, Aldibbiat A, Griffin W, Cox MA, Leech NJ, Shaw JA. A randomized pilot study in Type 1 diabetes complicated by severe hypoglycaemia, comparing rigorous hypoglycaemia avoidance with insulin analogue therapy, CSII or education alone. Diabet Med 2007; 24: 778-83.

5. Misso ML, Egberts KJ, Page M, O’Connor D, Shaw J. Continuous subcutaneous insulin infusion (CSII) versus multiple insulin injections for type 1 diabetes mellitus. Cochrane Database Syst Rev 2010; 20: CD005103.

6. Monami M, Lamanna C, Marchionni N, Mannucci E. Continuous subcutaneous insulin infusion versus multiple daily insulin injections in type 1 diabetes: a meta-analysis. Acta Diabetol 2010; 47 Suppl 1: 77-81.

7. National Institute for Health and Clinical Excellence. Continuous Subcutaneous Insulin Infusion for the Treatment of Diabetes Mellitus. Technology Appraisal Guidance 151 (Review of Technology Appraisal Guidance 57). NICE, London; 2008.

8. Grunberger G, Bailey TS, Cohen AJ, Flood TM, Handelsman Y. AACE Insulin Pump Management Task Force, et al. Statement by the American Association of Clinical Endocrinologists Consensus Panel on insulin pump management. Endocr Pract 2010; 16: 746-62.

9. Weissberg-Benchell J, Antisdel-Lomaglio J, Seshadri R. Insulin pump therapy: a meta-analysis. Diabetes Care 2003; 26: $1079-87$.
10. Sullivan-Bolyai S, Knafl K, Tamborlane W, Grey M. Parents' reflections on managing their children's diabetes with insulin pumps. J Nurs Scholarsh 2004; 36: 316 -23.

11. Hanas R, Ludvigsson J. Hypoglycemia and ketoacidosis with insulin pump therapy in children and adolescents. Pediatr Diabetes 2006; 7 (Suppl. 4): 32-38.

12. Phillip M, Battelino T, Rodriguez H, Danne T, Kaufman F; European Society for Paediatric Endocrinology; Lawson Wilkins Pediatric Endocrine Society; International Society for Pediatric and Adolescent Diabetes; American Diabetes Association; European Association for the Study of Diabetes. Use of insulin pump therapy in the paediatric age-group: consensus statement from the European Society for Paediatric Endocrinology, the Lawson Wilkins Pediatric Endocrine Society, and the International Society for Pediatric and Adolescent Diabetes, endorsed by the American Diabetes Association and the European Association for the Study of Diabetes. Diabetes Care 2007; 30: 1653-62.

13. Margeirsdottir HD, Larsen JR, Brunborg C, Dahl-Jorgensen K. Nationwide improvement in HbA1c and complication screening in a benchmarking project in childhood diabetes (Abstract). Pediatr Diabetes 2006; 7 (Suppl. 5): 18.

14. Farrar D, Tuffnell DJ, West J. Continuous subcutaneous insulin infusion versus multiple daily injections of insulin for pregnant women with diabetes. Cochrane Database Syst Rev 2007; 3: CD005542.

15. Mukhopadhyay A, Farrell T, Fraser RB, Ola B. Continuous subcutaneous insulin infusion vs intensive conventional insulin therapy in pregnant diabetic women: a systematic review and metaanalysis of randomized, controlled trials. Am J Obstet Gynecol 2007; 197: 447-56.

16. Pickup JC. Management of diabetes mellitus: is the pump mightier than the pen? Nat Rev Endocrinol 2012; 8: 425-33.

17. Simmons D, Thompson CF, Conroy C, Scott DJ. Use of insulin pumps in pregnancies complicated by type 2 diabetes and gestational diabetes in a multiethnic community. Diabetes Care 2001; 24: 2078-82.

18. Lauritzen T, Pramming S, Deckert T, Binder C. Pharmacokinetics of continuous subcutaneous insulin infusion. Diabetologia 1983; 24: 326-9.

19. Pickup JC. Insulin-pump therapy for type 1 diabetes mellitus. N Engl J Med 2012; 366: 1616-24. 